\title{
A MARCINKIEWICZ INTEGRAL TYPE CHARACTERIZATION OF THE SOBOLEV SPACE
}

\author{
PIOTR HAJŁASZ AND ZHUOMIN LIU
}

\begin{abstract}
In this paper we present a new characterization of the Sobolev space $W^{1, p}$, $1<p<\infty$ which is a higher dimensional version of a result of Waterman 32. We also provide a new and simplified proof of a recent result of Alabern, Mateu and Verdera [2]. Finally, we generalize the results to the case of weighted Sobolev spaces with respect to a Muckenhoupt weight.
\end{abstract}

\section{INTRODUCTION}

In connection with differentiability properties of periodic functions Marcinkiewicz [21] introduced the following integral

$$
\nu(f)=\left(\int_{0}^{2 \pi}|F(x+t)+F(x-t)-2 F(x)|^{2} \frac{d t}{t^{3}}\right)^{1 / 2}, \quad \text { where } \quad F(x)=\int_{0}^{x} f(t) d t .
$$

For more details regarding Marcinkiewicz's results see Vol. II, Chapter XIV, Theorems 5.1 and 5.3 in [36. Marcinkiewicz conjectured that for $1<p<\infty$ there is a constant $C_{p}>0$ such that

$$
\|\nu(f)\|_{p} \leq C_{p}\|f\|_{p} \quad \text { for } f \in L^{p}\left(S^{1}\right)
$$

and

$$
\|f\|_{p} \leq C_{p}\|\nu(f)\|_{p} \quad \text { for } f \in L^{p}\left(S^{1}\right) \text { such that } \int_{0}^{2 \pi} f(t) d t=0 .
$$

The condition in the second inequality that the integral vanishes is necessary, because for constant functions the right hand side of the inequality equals zero. The conjecture of Marcinkiewicz was answered in the affirmative by Zygmund [35]. Later Waterman [32] extended the method of Zygmund to the non-periodic case and he proved

Theorem 1.1. For $1<p<\infty$, there is a constant $C_{p} \geq 1$ such that

$$
C_{p}^{-1}\|f\|_{p} \leq\|\mu(f)\|_{p} \leq C_{p}\|f\|_{p}, \quad \text { for all } f \in L^{p}(\mathbb{R}),
$$

where

$$
\mu(f)(x)=\left(\int_{0}^{\infty}|F(x+t)+F(x-t)-2 F(x)|^{2} \frac{d t}{t^{3}}\right)^{1 / 2}, \quad F(x)=\int_{0}^{x} f(t) d t .
$$

2010 Mathematics Subject Classification. Primary 46E35; Secondary 42B25.

Key words and phrases. Sobolev spaces, Littlewood-Paley theory.

P.H. was supported by NSF grant DMS-1161425. Z.L. was supported by the ERC CZ grant LL1203 of the Czech Ministry of Education. 
Stein [26] generalized the Marcinkiewicz integral (1.1) to higher dimensions as follows. Let $\Omega \in L^{1}\left(S^{n-1}\right)$ have vanishing integral

$$
\int_{S^{n-1}} \Omega(y) d \sigma(y)=0 .
$$

The Marcinkiewicz integral of Stein is defined by

$$
\mu_{\Omega}(f)(x)=\left(\int_{0}^{\infty}\left|\int_{|y| \leq t} \frac{\Omega\left(y^{\prime}\right)}{|y|^{n-1}} f(x-y) d y\right|^{2} \frac{d t}{t^{3}}\right)^{1 / 2}, \quad \text { where } y^{\prime}=y /|y| .
$$

If $n=1$ and $\Omega\left(y^{\prime}\right)=\operatorname{sign} y$, we obtain integral (1.1). Stein proved in all dimensions that if $\Omega$ is odd, then $\mu_{\Omega}$ is bounded in $L^{p}, 1<p<\infty$, and if $\Omega$ is Hölder continuous with exponent $0<\alpha \leq 1$, then $\mu_{\Omega}$ is bounded in $L^{p}, 1<p \leq 2$, and is of weak type $(1,1)$. In the odd case the result was obtained as a consequence of the one dimensional result due to Waterman. The methods used by Stein were quite difficult. Later Benedek, Calderón and Panzone [3] proved the following result by way of vector valued singular integrals.

Theorem 1.2. If $\Omega \in C^{1}\left(S^{n-1}\right)$ satisfies (1.2), then for $1<p<\infty$ there is a constant $C=C(n, p) \geq 1$ such that

$$
\left\|\mu_{\Omega}(f)\right\|_{p} \leq C\|f\|_{p} \quad \text { for } f \in L^{p}\left(\mathbb{R}^{n}\right) .
$$

An optimal condition under which (1.4) is satisfied was discovered in [1]: (1.4) holds true provided $\Omega$ satisfies (1.2) and $\Omega \in L(\log L)^{1 / 2}\left(S^{n-1}\right)$. A generalization of Theorem 1.2 to the case of weighted $L_{w}^{p}\left(\mathbb{R}^{n}\right)$ spaces, where $w \in A_{p}$ is a Muckenhoupt weight, was obtained by Sato in [24], see Theorem 4.2 below. For recent sharp results, see [7]. There has been a tremendous development of the theory of Marcinkiewicz integrals (literally hundreds of papers) and it is simply not possible to provide relevant references here; nonetheless the reader will have no problems with finding them.

It turns out that under certain additional assumptions about $\Omega$ we have

$$
C^{-1}\|f\|_{p} \leq\left\|\mu_{\Omega}(f)\right\|_{p} \leq C\|f\|_{p} \text { for } f \in L^{p}\left(\mathbb{R}^{n}\right) .
$$

The left inequality is obtained from the right one by a duality argument (see Step 4 in Section 3 for details). These assumptions are satisfied for example by $\Omega\left(y^{\prime}\right)=\operatorname{sign} y$ when $n=1$ and hence Theorem 1.1 follows.

Recall that the Sobolev space $W^{1, p}\left(\mathbb{R}^{n}\right)$ is the space of functions $f \in L^{p}\left(\mathbb{R}^{n}\right)$ with first order weak derivatives in $L^{p}\left(\mathbb{R}^{n}\right) . W^{1, p}\left(\mathbb{R}^{n}\right)$ is a Banach space with the norm $\|f\|_{1, p}=$ $\|f\|_{p}+\|\nabla f\|_{p}$. Observe that Theorem 1.1 can be regarded as a characterization of the Sobolev space $W^{1, p}(\mathbb{R})$. Indeed, if $f \in W^{1, p}(\mathbb{R}), 1<p<\infty$, and

$$
T(f)=\left(\int_{0}^{\infty}|f(x+t)+f(x-t)-2 f(x)|^{2} \frac{d t}{t^{3}}\right)^{1 / 2},
$$

then $T(f)=\mu\left(f^{\prime}\right)$ and hence

$$
C_{p}^{-1}\left\|f^{\prime}\right\|_{p} \leq\|T(f)\|_{p} \leq C_{p}\left\|f^{\prime}\right\|_{p} .
$$

It follows from this inequality that $f \in W^{1, p}(\mathbb{R})$ if and only if $f \in L^{p}(\mathbb{R})$ and $T(f) \in L^{p}(\mathbb{R})$. Stein [27], [28, p. 163] generalized this characterization to higher dimensions as follows 
Theorem 1.3. $f \in W^{1, p}\left(\mathbb{R}^{n}\right), \frac{2 n}{n+1}<p<\infty$ if and only if $f \in L^{p}\left(\mathbb{R}^{n}\right)$ and

$$
\left(\int_{\mathbb{R}^{n}} \frac{|f(x+y)+f(x-y)-2 f(x)|^{2}}{|y|^{n+2}} d y\right)^{1 / 2} \in L^{p}\left(\mathbb{R}^{n}\right) .
$$

Note that when $n=1$, the integral in (1.8) equals $\sqrt{2} T(f)$ and hence Theorem 1.3 is a natural generalization of the characterization of $W^{1, p}(\mathbb{R})$ mentioned above to higher dimensions, but a problem is that in higher dimensions Theorem 1.3 does not cover the case $1<p \leq 2 n /(n+1)$. Actually Stein proved a more general result that includes a characterization of Bessel potential spaces. For other related characterizations of Sobolev and Bessel potential spaces, see for example [2, 6, 22, 30, 33, 34]. We will discus the paper [2] later on.

One of the aims of this paper is to generalize the characterization (1.7) to higher dimensions in a way that it would be valid for all $1<p<\infty$. To avoid the limitation on the exponent $p$ in Stein's Theorem 1.3, we generalize the Marcinkiewicz integral in a different way. Observe that in dimension one

$$
\frac{f(x+t)+f(x-t)-2 f(x)}{2}=f_{S(x, t)} f(y) d \sigma(y)-f(x)=f_{S(x, t)}-f(x),
$$

where the barred integral denotes the integral average and in our case we take the average over the zero dimensional sphere $S(x, t)$. Here and in what follows $f_{E}$ is used to denote the integral average of $f$ over $E$. Now for $f \in L^{p}\left(\mathbb{R}^{n}\right)$ we define

$$
T f(x)=\left(\int_{0}^{\infty}\left|f(x)-f_{S(x, t)}\right|^{2} \frac{d t}{t^{3}}\right)^{1 / 2} .
$$

Note that when $n=1$, the definition (1.9) is consistent with (1.6) (up to a constant factor). One of the main results of this paper reads as follows.

Theorem 1.4. Suppose that $f \in L^{p}\left(\mathbb{R}^{n}\right), 1<p<\infty$. Then $f \in W^{1, p}\left(\mathbb{R}^{n}\right)$ if and only if Tf $\in L^{p}\left(\mathbb{R}^{n}\right)$. Moreover there is a constant $C=C(n, p) \geq 1$ such that

$$
C^{-1}\|\nabla f\|_{p} \leq\|T f\|_{p} \leq C\|\nabla f\|_{p}
$$

When $n=1$, Theorem 1.4 is the same as the characterization (1.7). We will also prove that $T f$ can be expressed as a Marcinkiewicz integral (1.3) of $\nabla f$. Let

$$
\phi(x)=\frac{1}{n \omega_{n}} \frac{x}{|x|^{n}} \chi_{B(0,1)}(x) \quad \text { and } \quad \phi_{t}(x)=t^{-n} \phi(x / t)=\frac{1}{\operatorname{tn} \omega_{n}} \frac{x}{|x|^{n}} \chi_{B(0, t)}(x) .
$$

Here and in what follows $\omega_{n}$ is the volume of a unit ball in $\mathbb{R}^{n}$ and hence $n \omega_{n}$ is the surface area of the sphere $S^{n-1}(0,1)$.

Lemma 1.5. If $f \in W_{\mathrm{loc}}^{1,1}\left(\mathbb{R}^{n}\right)$, then

$$
T f(x)=\left(\int_{0}^{\infty}\left|\phi_{t} * \nabla f(x)\right|^{2} \frac{d t}{t}\right)^{1 / 2}=\frac{1}{n \omega_{n}}\left(\int_{0}^{\infty}\left|\int_{|y| \leq t} \frac{y}{|y|^{n}} \cdot \nabla f(x-y) d y\right|^{2} \frac{d t}{t^{3}}\right)^{1 / 2}
$$

for almost all $x \in \mathbb{R}^{n}$. 
The functions $\phi_{t}$ and $\nabla f$ take values in $\mathbb{R}^{n}$ and $\phi_{t} * \nabla f$ is defined as the integral of the scalar product

$$
\phi_{t} * \nabla f(x)=\int_{\mathbb{R}^{n}} \phi_{t}(y) \cdot \nabla f(x-y) d y .
$$

The inequality $\|T f\|_{p} \leq C\|\nabla f\|_{p}$ follows directly from Lemma 1.5 and Theorem 1.2, The proof of the reverse inequality $\|\nabla f\|_{p} \leq C\|T f\|_{p}$ will be obtained by a standard duality argument; see Section 3, Steps 4 and 5.

Theorem 1.1 can be regarded as a characterization of the Sobolev space $W^{1, p}(\mathbb{R})$, see (1.7) and a comment that follows. Theorems 1.2 and 1.3 are higher dimensional generalizations of Theorem 1.1. However, in higher dimensions Theorem 1.2 cannot be interpreted as a characterization of $W^{1, p}\left(\mathbb{R}^{n}\right)$ and in Theorem 1.3 we can characterize $W^{1, p}\left(\mathbb{R}^{n}\right)$ but only for $p>2 n /(n+1)$. From this perspective Theorem 1.4 is a more natural generalization on Theorem 1.1 to higher dimensions: it works for all $1<p<\infty$ and it gives a characterization of $W^{1, p}\left(\mathbb{R}^{n}\right)$ in terms of the Marcinkiewicz integral of the gradient (1.11), just like the characterization (1.7) in dimension $n=1$.

Theorem 1.4 is related to a recent characterization of $W^{1, p}\left(\mathbb{R}^{n}\right)$ due to Alabern, Mateu and Verdera [2, Theorem 1] where instead of subtracting averages over spheres we subtract averages over balls.

Theorem 1.6. Suppose that $f \in L^{p}\left(\mathbb{R}^{n}\right), 1<p<\infty$. Then $f \in W^{1, p}\left(\mathbb{R}^{n}\right)$ if and only if $S f \in L^{p}\left(\mathbb{R}^{n}\right)$, where

$$
S f(x)=\left(\int_{0}^{\infty}\left|f(x)-f_{B(x, t)}\right|^{2} \frac{d t}{t^{3}}\right)^{1 / 2} .
$$

Moreover there is a constant $C=C(n, p) \geq 1$ such that

$$
C^{-1}\|\nabla f\|_{p} \leq\|S f\|_{p} \leq C\|\nabla f\|_{p}
$$

We will provide a new, and on a technical side much simpler, proof of this result based on the following representation formula. Let

$$
\psi(x)=\frac{1}{n \omega_{n}}\left(\frac{x}{|x|^{n}}-x\right) \chi_{B(0,1)}(x) \quad \text { and } \quad \psi_{t}(x)=t^{-n} \psi(x / t) .
$$

Lemma 1.7. If $f \in W_{\mathrm{loc}}^{1,1}\left(\mathbb{R}^{n}\right)$, then

$S f(x)=\left(\int_{0}^{\infty}\left|\psi_{t} * \nabla f(x)\right|^{2} \frac{d t}{t}\right)^{1 / 2}=\frac{1}{n \omega_{n}}\left(\int_{0}^{\infty}\left|\int_{|y| \leq t}\left(\frac{y}{|y|^{n}}-\frac{y}{t^{n}}\right) \cdot \nabla f(x-y) d y\right|^{2} \frac{d t}{t^{3}}\right)^{1 / 2}$

for almost all $x \in \mathbb{R}^{n}$.

The original proof of Theorem [1.6 is based on vector valued singular integrals and the main technical difficulty is a verification that a suitable vector valued integral operator satisfies the Hörmander condition. Our approach is much simpler as we will show that the inequality $\|S f\|_{p} \leq C\|\nabla f\|_{p}$ in Theorem 1.6 is a direct consequence of Lemma 1.7. Theorem 1.2, and the following classical result in the Littlewood-Paley theory due to Benedek, Calderón and Panzone [3], [31, Chapter XII, Theorem 3.5]. (Alternatively one can use a result of Sato, Theorem 4.2, in place of Theorems 1.2 and 1.8, see (4.1).) 
Theorem 1.8. Let $\phi \in L^{1}\left(\mathbb{R}^{n}\right)$ be such that

$$
\int_{\mathbb{R}^{n}} \phi(x) d x=0 .
$$

Assume that there are constants $C, \alpha>0$ such that

$$
|\phi(x)| \leq C(1+|x|)^{-n-\alpha}, \quad x \in \mathbb{R}^{n}
$$

and

$$
\int_{\mathbb{R}^{n}}|\phi(x+h)-\phi(x)| d x \leq C|h|^{\alpha}, \quad h \in \mathbb{R}^{n} .
$$

Let $\phi_{t}(x)=t^{-n} \phi(x / t)$. Then the operator

$$
G f(x)=\left(\int_{0}^{\infty}\left|\phi_{t} * f(x)\right|^{2} \frac{d t}{t}\right)^{1 / 2}
$$

is bounded in $L^{p}, 1<p<\infty$ and of weak type $(1,1)$.

The main result of [2, Theorem 3] is actually more general since it also covers the case of higher order derivatives and the case of Bessel potential spaces. It is possible to modify Theorem 1.4 in a way that it would cover the case of higher order derivatives, but we decided to restrict to the case of the first order derivatives for the sake of simplicity.

It is interesting to point out that the functions $S f$ and $T f$ satisfy the following pointwise inequality.

Proposition 1.9. If $f \in W^{1, p}\left(\mathbb{R}^{n}\right), 1 \leq p \leq \infty$, then

$$
S f(x) \leq \frac{n}{n+2} T f(x) \quad \text { a.e. }
$$

Actually it follows from the proof that (1.16) holds true under a weaker assumption that $f \in W_{\mathrm{loc}}^{1,1}\left(\mathbb{R}^{n}\right)$ is such that

$$
\lim _{t \rightarrow \infty} \frac{1}{t} f_{B(x, t)}|f(y)| d y=0 .
$$

As we pointed out, the inequality $\|T f\|_{p} \leq C\|\nabla f\|_{p}$ is a direct consequence of Theorem 1.2 and the elementary formula (1.11). This combined with Proposition 1.9 proves also the inequality $\|S f\|_{p} \leq C\|\nabla f\|_{p}$, but the proof of the reverse inequality $\|\nabla f\|_{p} \leq C\|S f\|_{p}$ cannot be directly concluded from Theorem 1.4 and Proposition 1.9. To prove the reverse inequality we will use Lemma 1.7 instead of Proposition [1.9, For this reason we will prove Theorem 1.6 directly without referring to Proposition [1.9. We will prove Proposition 1.9 in Section 5, after the proofs of Theorems 1.4 and 1.6 since it will not be used in these proofs.

We believe that the content of this paper will be of interest mostly for the community of people working with geometric aspects of Sobolev spaces. Since many of the researchers working in this area do not use tools form harmonic analysis, we decided to make the paper self-contained and easy to read by providing all necessary details. But we also hope that researchers whose main area of research is harmonic analysis will find this paper interesting too. 
Notation used in the paper is pretty standard. The Fourier transform is defined by

$$
\hat{f}(\xi)=\int_{\mathbb{R}^{n}} e^{-2 \pi i x \cdot \xi} f(x) d x .
$$

By $C$ we will denote a positive constant whose value may change in a single string of estimates.

The paper is organized as follows. In Section 2 we prove Lemmas 1.5 and 1.7. The proofs are very elementary. In Section 3 we prove Theorems 1.4 and 1.6. The proofs use some harmonic analysis including Theorems 1.2 and 1.8. In Section 4 we prove the second main result of the paper, Theorem 4.1 which is a generalization of Theorems 1.4 and 1.6 to the case of weighted Sobolev spaces with a Muckenhoupt weight. Theorems 1.4 and 1.6]are special cases of Theorem 4.1, but we decided to include separate proofs in the unweighted case, because the proofs are based on more elementary arguments (in particular we could use classical Theorems 1.2 and 1.8 in place of a more complicated Theorem 4.2) and the proofs of Theorems 1.4 and 1.6 are in fact used in the proof of Theorem 4.1. Proposition 1.9 which gives an inequality between $S f$ and $T f$ is presented in Section 5. This result is not needed in the proofs of Theorems 1.4, 1.6, and 4.1. In Section 6 we include final remarks which are of independent interest - they are not needed in the proofs of the results in the earlier sections.

Acknowledgments. We would like to thank Yibiao Pan and Joan Verdera for helpful discussions. Yibiao Pan showed us that the duality argument works also in the weighted case which was needed to complete the proof of Theorem 4.1. We would also like to thank Shuichi Sato for providing a copy of his very recent work [23], where some of the results of this paper have been generalized.

\section{Proof of Lemmas 1.5 and 1.7}

Both of the lemmas follow immediately from the lemma below.

Lemma 2.1. If $f \in W_{\mathrm{loc}}^{1,1}\left(\mathbb{R}^{n}\right)$, then for all $t>0$ and almost all $x \in \mathbb{R}^{n}$ we have

$$
\begin{gathered}
f(x)-f_{S(x, t)}=\frac{1}{n \omega_{n}} \int_{B(x, t)} \nabla f(y) \cdot \frac{x-y}{|x-y|^{n}} d y, \\
f(x)-f_{B(x, t)}=\frac{1}{n \omega_{n}} \int_{B(x, t)} \nabla f(y) \cdot \frac{x-y}{|x-y|^{n}} d y-\frac{1}{n} f_{B(x, t)} \nabla f(y) \cdot(x-y) d y .
\end{gathered}
$$

Proof. We can assume that $f \in C_{0}^{\infty}\left(\mathbb{R}^{n}\right)$. The general case will follow by approximation. Note that the restriction (trace) of $f \in W_{\text {loc }}^{1,1}$ to $S(x, t)$ is well defined [8, Section 4.3, 
Theorem 1]. We have

$$
\begin{aligned}
f(x)-f_{S(x, t)} f(y) d \sigma(y) & =-\int_{0}^{t} \frac{d}{d \tau}\left(f_{S(x, \tau)} f(y) d \sigma(y)\right) d \tau \\
& =-\int_{0}^{t} \frac{d}{d \tau}\left(f_{S(0,1)} f(x+\tau z) d \sigma(z)\right) d \tau \\
& =-\int_{0}^{t} f_{S(0,1)} \nabla f(x+\tau z) \cdot z d \sigma(z) d \tau \\
& =-\int_{0}^{t} f_{S(x, \tau)} \nabla f(y) \cdot \frac{y-x}{|y-x|} d \sigma(y) d \tau \\
& =\frac{1}{n \omega_{n}} \int_{0}^{t} \int_{S(x, \tau)} \nabla f(y) \cdot \frac{x-y}{|x-y|^{n}} d \sigma(y) d \tau \\
& =\frac{1}{n \omega_{n}} \int_{B(x, t)} \nabla f(y) \cdot \frac{x-y}{|x-y|^{n}} d y .
\end{aligned}
$$

This proves the first identity. The proof of the second one is similar.

$$
\begin{aligned}
f(x)-f_{B(x, t)} f(y) d y & =-\int_{0}^{t} \frac{d}{d \tau}\left(f_{B(x, \tau)} f(y) d y\right) d \tau \\
& =-\int_{0}^{t} \frac{d}{d \tau}\left(f_{B(0,1)} f(x+\tau z) d z\right) d \tau \\
& =-\int_{0}^{t} f_{B(0,1)} \nabla f(x+\tau z) \cdot z d z d \tau \\
& =-\int_{0}^{t} f_{B(x, \tau)} \nabla f(y) \cdot \frac{y-x}{\tau} d y d \tau \\
& =\frac{1}{\omega_{n}} \int_{0}^{t} \int_{B(x, \tau)} \nabla f(y) \cdot \frac{x-y}{\tau^{n+1}} d y d \tau \\
& =\frac{1}{\omega_{n}} \int_{B(x, t)}\left(\int_{|x-y|}^{t} \frac{d \tau}{\tau^{n+1}}\right) \nabla f(y) \cdot(x-y) d y \\
& =\frac{1}{n \omega_{n}} \int_{B(x, t)}\left(\frac{1}{|x-y|^{n}}-\frac{1}{t^{n}}\right) \nabla f(y) \cdot(x-y) d y .
\end{aligned}
$$

The proof is complete.

\section{Proof of Theorems 1.4 And 1.6}

Step 1. $\|T f\|_{p} \leq C\|\nabla f\|_{p}$ and $\|S f\|_{p} \leq C\|\nabla f\|_{p}$.

As we already pointed out the inequality $\|T f\|_{p} \leq C\|\nabla f\|_{p}$ for $f \in W^{1, p}$ follows directly from Theorem 1.2 and Lemma 1.5. To prove the inequality $\|S f\|_{p} \leq C\|\nabla f\|_{p}$ observe that

$$
\psi(x)=\phi(x)-\eta(x), \quad \text { where } \quad \eta(x)=\frac{x}{n \omega_{n}} \chi_{B(0,1)}
$$


Hence

$$
S f(x) \leq T f(x)+W f(x)
$$

where

$$
W f(x)=\left(\int_{0}^{\infty}\left|\eta_{t} * \nabla f(x)\right|^{2} \frac{d t}{t}\right)^{1 / 2}
$$

It remains to show that $\|W f\|_{p} \leq C\|\nabla f\|_{p}, f \in W^{1, p}$. This is however, a consequence of Theorem 1.8. Indeed, the function $\eta$ clearly satisfies (1.13) and (1.14) with $\alpha=1$ and condition (1.15) also holds with $\alpha=1$ which can be justified as follows.

If $|h|>1 / 2$, then

$$
\int_{\mathbb{R}^{n}}|\eta(x+h)-\eta(x)| d x \leq 2\|\eta\|_{1} \leq C|h|
$$

If $|h| \leq 1 / 2$, then

$$
n \omega_{n} \int_{\mathbb{R}^{n}}|\eta(x+h)-\eta(x)| d x \leq \int_{B(0,1+|h|) \backslash B(0,1-|h|)} 2 d x+\int_{B(0,1-|h|)}|h| d x \leq C|h| .
$$

Step 2. Square functions $\tilde{T} g$ and $\tilde{S} g$.

In this subsection we modify the definitions of the square functions $T f$ and $S f$ and prove boundedness of these modified square functions in $L^{p}$. It will play a crucial role in the proof of the reverse inequalities $\|\nabla f\|_{p} \leq\|T f\|_{p}$ and $\|\nabla f\|_{p} \leq\|S f\|_{p}$.

For $g \in L^{p}\left(\mathbb{R}^{n}\right)$ let

$$
R g=\left(R_{1} g, \ldots, R_{n} g\right)
$$

be the vector valued Riesz transform, where

$$
(R \varphi)^{\wedge}(\xi)=-i \frac{\xi}{|\xi|} \hat{\varphi}(\xi) \quad \text { for } \varphi \in \mathscr{S}\left(\mathbb{R}^{n}\right) .
$$

Now we define

$$
\begin{aligned}
& \tilde{T} g(x)=\left(\int_{0}^{\infty}\left|\phi_{t} * R g(x)\right|^{2} \frac{d t}{t}\right)^{1 / 2}, \quad g \in L^{p}\left(\mathbb{R}^{n}\right), \\
& \tilde{S} g(x)=\left(\int_{0}^{\infty}\left|\psi_{t} * R g(x)\right|^{2} \frac{d t}{t}\right)^{1 / 2}, \quad g \in L^{p}\left(\mathbb{R}^{n}\right) .
\end{aligned}
$$

Lemma 3.1. For $1<p<\infty$ we have

$$
\begin{aligned}
& \|\tilde{T} g\|_{p} \leq C\|g\|_{p}, \quad g \in L^{p}\left(\mathbb{R}^{n}\right), \\
& \|\tilde{S} g\|_{p} \leq C\|g\|_{p}, \quad g \in L^{p}\left(\mathbb{R}^{n}\right) .
\end{aligned}
$$

Proof. Estimate (3.2) follows from Theorem 1.2 and boundedness of the Riesz transform in $L^{p}$ while (3.3) follows from

$$
\tilde{S} g \leq \tilde{T} g+\left(\int_{0}^{\infty}\left|\eta_{t} * R g\right|^{2} \frac{d t}{t}\right)^{1 / 2}
$$

combined with (3.2), the fact that $\eta$ satisfies the assumptions of Theorem 1.8 and from boundedness of the Riesz transform in $L^{p}$. 
Step 3. $L^{2}$ isometries.

We will prove that up to a constant factor, the square functions $\tilde{T}$ and $\tilde{S}$ are isometries in $L^{2}$, i.e.

Lemma 3.2. There are constants $C_{1}, C_{2}>0$ such that

$$
\|\tilde{T} g\|_{2}=C_{1}\|g\|_{2} \quad \text { and } \quad\|\tilde{S} g\|_{2}=C_{2}\|g\|_{2} \quad \text { for } g \in L^{2}\left(\mathbb{R}^{n}\right) .
$$

Observe that the functions $\phi$ and $\psi$ are of the form $g(|x|) x /|x|$. This allows us to find the structure of the Fourier transforms of $\phi$ and $\psi$.

Lemma 3.3. Let $f \in L^{1}\left(\mathbb{R}^{n}, \mathbb{R}^{n}\right)$ be of the form

$$
f(x)=\frac{x}{|x|} g(|x|) .
$$

Then there is a continuous function $h:[0, \infty) \rightarrow \mathbb{R}, h(0)=0, h(t) \rightarrow 0$ as $t \rightarrow \infty$ such that

$$
\hat{f}(\xi)=i \frac{\xi}{|\xi|} h(|\xi|), \quad \xi \in \mathbb{R}^{n} .
$$

The proof is based on the following well known result from linear algebra [12, Lemma 4.1.15], [28, p.57].

Lemma 3.4. If $m: \mathbb{R}^{n} \rightarrow \mathbb{R}^{n}$ is a measurable function that is homogeneous of degree 0 , i.e. $m(t x)=m(x)$ for $t>0$, and commutes with orthogonal transformations, i.e.

$$
m(\rho(x))=\rho(m(x)), \quad x \in \mathbb{R}^{n}, \rho \in O(n),
$$

then there is a constant $C \in \mathbb{R}$ such that

$$
m(x)=C \frac{x}{|x|} \text { for all } x \neq 0 .
$$

Proof of Lemma 3.3. Since the function $f$ is odd and takes values in $\mathbb{R}^{n}$, the real part of $\hat{f}$ equals zero, and hence

$$
i \hat{f}(\xi)=\int_{\mathbb{R}^{n}} \sin (2 \pi x \cdot \xi) f(x) d x
$$

takes values in $\mathbb{R}^{n}$. Fix $k>0$ and define $m_{k}: S^{n-1}(0, k) \rightarrow \mathbb{R}^{n}$ by $m_{k}(\xi)=i \hat{f}(\xi)$ for $|\xi|=k$. Extend $m_{k}$ to $m_{k}: \mathbb{R}^{n} \backslash\{0\} \rightarrow \mathbb{R}^{n}$ as a function homogeneous of degree 0 , i.e.

$$
m_{k}(\xi)=i \hat{f}\left(\frac{k \xi}{|\xi|}\right) \quad \text { for } \xi \neq 0 .
$$

We claim that

$$
m_{k}(\rho(\xi))=\rho\left(m_{k}(\xi)\right) \quad \text { for } \rho \in O(n) \text { and } \xi \neq 0 .
$$


Indeed, it suffices to check (3.4) for $|\xi|=k$. We have

$$
\begin{aligned}
m_{k}(\rho(\xi)) & =\int_{\mathbb{R}^{n}} \sin (2 \pi x \cdot \rho(\xi)) \frac{x}{|x|} g(|x|) d x=\int_{\mathbb{R}^{n}} \sin \left(2 \pi \rho^{-1}(x) \cdot \xi\right) \frac{x}{|x|} g(|x|) d x \\
& =\int_{\mathbb{R}^{n}} \sin (2 \pi x \cdot \xi) \frac{\rho(x)}{|\rho(x)|} g(|\rho(x)|) d x=\int_{\mathbb{R}^{n}} \sin (2 \pi x \cdot \xi) \frac{\rho(x)}{|x|} g(|x|) d x \\
& =\rho\left(\int_{\mathbb{R}^{n}} \sin (2 \pi x \cdot \xi) \frac{x}{|x|} g(|x|) d x\right)=\rho\left(m_{k}(\xi)\right) .
\end{aligned}
$$

According to Lemma 3.4 there is a constant $h(k) \in \mathbb{R}$ such that $m_{k}(\xi)=-h(k) \xi /|\xi|$. In particular for $|\xi|=k$ we have

$$
i \hat{f}(\xi)=m_{k}(\xi)=-\frac{\xi}{|\xi|} h(|\xi|) .
$$

Clearly $h$ is continuous, $h(0)=0$ and $h(t) \rightarrow 0$ as $t \rightarrow \infty$, because $\hat{f} \in C_{0}\left(\mathbb{R}^{n}, \mathbb{R}^{n}\right)$.

Proof of Lemma 3.2. We will prove the result in the case of the square function $\tilde{T} g$ only. The proof in the case of $\tilde{S} g$ is the same. Using the Fubini theorem, the Plancherel theorem and the fact that $\hat{\phi}_{t}(\xi)=\hat{\phi}(t \xi)$ we obtain

$$
\begin{gathered}
\|\tilde{T} g\|_{2}^{2}=\int_{0}^{\infty} \int_{\mathbb{R}^{n}}\left|\phi_{t} * R g\right|^{2} d x \frac{d t}{t}=\int_{0}^{\infty} \int_{\mathbb{R}^{n}}|\hat{\phi}(t \xi) \cdot \widehat{R g}(\xi)|^{2} d \xi \frac{d t}{t}=\varnothing, \\
\hat{\phi}(t \xi)=i \frac{\xi}{|\xi|} h(t|\xi|), \quad \widehat{R g}(\xi)=-i \frac{\xi}{|\xi|} \hat{g}(\xi), \\
\varnothing=\int_{0}^{\infty} \int_{\mathbb{R}^{n}}|\hat{g}(\xi) h(t|\xi|)|^{2} d \xi \frac{d t}{t}=\int_{\mathbb{R}^{n}}|g(x)|^{2} d x \int_{0}^{\infty}|h(t)|^{2} \frac{d t}{t}=C\|g\|_{2}^{2} .
\end{gathered}
$$

Note that the integral involving $h$ does not depend on $|\xi|$ (use the change of variables $s=t|\xi|)$. Since the square function $\tilde{T} g$ is bounded in $L^{2}$ we conclude that

$$
\int_{0}^{\infty}|h(t)|^{2} \frac{d t}{t}=C<\infty .
$$

The proof is complete.

Step 4. Duality argument.

We will use a standard duality argument [9, Remark 5.6, p. 507], [10, Exercise 5.1.6], to show that Lemma 3.1 and Lemma 3.2 imply

Lemma 3.5. For $1<p<\infty$ there is a constant $C \geq 1$ such that

$$
\begin{array}{ll}
C^{-1}\|g\|_{p} \leq\|\tilde{T} g\|_{p} \leq C\|g\|_{p}, & g \in L^{p}\left(\mathbb{R}^{n}\right), \\
C^{-1}\|g\|_{p} \leq\|\tilde{S} g\|_{p} \leq C\|g\|_{p}, & g \in L^{p}\left(\mathbb{R}^{n}\right) .
\end{array}
$$

Proof. We will prove the result in the $\tilde{T} g$ case, the proof in the $\tilde{S} g$ case is the same. Consider the following operator acting on functions $g$ defined on $\mathbb{R}^{n}$ whose values at $x \in \mathbb{R}^{n}$ are measurable functions of variable $t \in \mathbb{R}_{+}$

$$
K g(x)=\left(\phi_{t} * R g(x)\right)_{t>0} .
$$


Lemma 3.1 states that $K$ is a bounded operator between the spaces

$$
K: L^{p}\left(\mathbb{R}^{n}\right) \rightarrow L^{p}\left(\mathbb{R}^{n}, L^{2}\left(\mathbb{R}_{+}, d t / t\right)\right)=L^{p}\left(\mathbb{R}^{n}, H\right),
$$

and Lemma 3.2 means that $K: L^{2}\left(\mathbb{R}^{n}\right) \rightarrow L^{2}\left(\mathbb{R}^{n}, H\right)$ is an isometry multiplied by a constant factor

$$
\left(\int_{\mathbb{R}^{n}}\|K g\|_{H}^{2} d x\right)^{1 / 2}=C_{1}\left(\int_{\mathbb{R}^{n}}|g|^{2} d x\right)^{1 / 2}, \quad g \in L^{2}\left(\mathbb{R}^{n}\right) .
$$

Here we consider real valued functions $g$. Let $q$ be the Hölder conjugate exponent to $p$, $p^{-1}+q^{-1}=1$. Since the scalar product is determined by the Hilbert norm (polarization identity) we conclude from (3.6) and from (3.2) with $p$ replaced by $q$ that for $g \in L^{p} \cap L^{2}$ and $h \in L^{q} \cap L^{2}$ we have

$$
\begin{aligned}
C_{1}^{2} \int_{\mathbb{R}^{n}} g h d x & =\int_{\mathbb{R}^{n}}\langle K g, K h\rangle_{H} d x \leq\left(\int_{\mathbb{R}^{n}}\|K g\|_{H}^{p} d x\right)^{1 / p}\left(\int_{\mathbb{R}^{n}}\|K h\|_{H}^{q} d x\right)^{1 / q} \\
& =\|\tilde{T} g\|_{p}\|\tilde{T} h\|_{q} \leq C\|\tilde{T} g\|_{p}\|h\|_{q} .
\end{aligned}
$$

Taking supremum over $h \in L^{q} \cap L^{2},\|h\|_{q} \leq 1$ we obtain $\|g\|_{p} \leq C\|\tilde{T} g\|_{p}$. We proved this inequality for $g \in L^{p} \cap L^{2}$, but a density argument shows that it is true for any $g \in L^{p}\left(\mathbb{R}^{n}\right)$.

Step 5. Fractional Laplacian $(-\Delta)^{1 / 2}$.

In this section we will prove the left inequalities at (1.10) and (1.12) for $f \in W^{1, p}$ by applying Lemma [3.5 to $g=(-\Delta)^{1 / 2} f$.

Recall that the fractional Laplace operator is defined by

$$
(-\Delta)^{1 / 2} \varphi=(2 \pi|\xi| \hat{\varphi}(\xi))^{\vee}, \quad \varphi \in \mathscr{S}\left(\mathbb{R}^{n}\right) .
$$

Lemma 3.6. For $1<p<\infty$ there is a constant $C \geq 1$ such that

$$
C^{-1}\|\nabla \varphi\|_{p} \leq\left\|(-\Delta)^{1 / 2} \varphi\right\|_{p} \leq C\|\nabla \varphi\|_{p}, \quad \varphi \in \mathscr{S}\left(\mathbb{R}^{n}\right) .
$$

Proof. By taking the Fourier transform and looking at the multipliers we see that

$$
R(-\Delta)^{1 / 2} \varphi=-\nabla \varphi \text { and } R \cdot \nabla \varphi=(-\Delta)^{1 / 2} \varphi,
$$

where $R \cdot \nabla \varphi=\sum_{j} R_{j} \partial_{j} \varphi$. Then the result follows from boundedness of $R$ in $L^{p}$.

By continuity $(-\Delta)^{1 / 2}$ uniquely extends to a bounded operator

$$
(-\Delta)^{1 / 2}: W^{1, p}\left(\mathbb{R}^{n}\right) \rightarrow L^{p}\left(\mathbb{R}^{n}\right), \quad 1<p<\infty
$$

that also satisfies the inequality of Lemma 3.6 and

$$
R(-\Delta)^{1 / 2} f=-\nabla f \quad \text { for } f \in W^{1, p}\left(\mathbb{R}^{n}\right), 1<p<\infty .
$$

Now for $f \in W^{1, p}\left(\mathbb{R}^{n}\right)$ Lemma 3.5 yields

$$
\|T f\|_{p}=\left\|\tilde{T}\left((-\Delta)^{1 / 2} f\right)\right\|_{p} \approx\left\|(-\Delta)^{1 / 2} f\right\|_{p} \approx\|\nabla f\|_{p}
$$

and similarly $\|S f\|_{p} \approx\|\nabla f\|_{p}$.

Step 6. The final step of the proof. 
We proved that $f \in W^{1, p}, 1<p<\infty$ satisfies the inequalities (1.10) and (1.12). It remains to prove that if $f \in L^{p}$ satisfies $T f \in L^{p}\left(\mathbb{R}^{n}\right)$ or $S f \in L^{p}\left(\mathbb{R}^{n}\right)$, then $f \in W^{1, p}$.

Let $f \in L^{p}\left(\mathbb{R}^{n}\right), 1<p<\infty$ and assume that $T f \in L^{p}$ or $S f \in L^{p}$. Observe that the functions $T f$ and $S f$ are of the form

$$
K f(x)=\left(\int_{0}^{\infty}\left|f * \mu_{t}(x)\right|^{2} \frac{d t}{t^{3}}\right)^{1 / 2},
$$

where $\mu_{t}$ is some measure. For example in the case of the square function $T f, \mu_{t}$ is the Dirac delta minus the Hausdorff measure $\mathcal{H}^{n-1}$ restricted to $S^{n-1}(0, t)$, normalized to have total mass 1.

Let $\varphi$ be a standard mollifier, i.e. $\varphi \in C_{0}^{\infty}\left(\mathbb{R}^{n}\right), \varphi \geq 0, \int_{\mathbb{R}^{n}} \varphi(x) d x=1$. Let $\varphi_{\varepsilon}(x)=$ $\varepsilon^{-n} \varphi(x / \varepsilon)$ and $f_{\varepsilon}=f * \varphi_{\varepsilon}$. Clearly $f_{\varepsilon} \rightarrow f$ in $L^{p}$. Since $\nabla f_{\varepsilon}=f * \nabla \varphi_{\varepsilon},\left\|\nabla f_{\varepsilon}\right\|_{p} \leq$ $\|f\|_{p}\left\|\nabla \varphi_{\varepsilon}\right\|_{1}<\infty$. Hence $f_{\varepsilon} \in W^{1, p}$ and thus

$$
\left\|K f_{\varepsilon}\right\|_{p} \approx\left\|\nabla f_{\varepsilon}\right\|_{p}
$$

On the other hand for almost all $x \in \mathbb{R}^{n}$ we have

$K f_{\varepsilon}(x)=\left(\int_{0}^{\infty}\left|\int_{\mathbb{R}^{n}}\left(f * \mu_{t}\right)(x-y) \varphi_{\varepsilon}(y) d y\right|^{2} \frac{d t}{t^{3}}\right)^{1 / 2} \leq\left(\int_{0}^{\infty}\left(\int_{\mathbb{R}^{n}}|F(y, t)| \varphi_{\varepsilon}(y) d y\right)^{2} \frac{d t}{t^{3}}\right)^{1 / 2}$,

where $F(y, t)=\left(f * \mu_{t}\right)(x-y)$. Thus the Minkowski integral inequality, [28], implies

$$
K f_{\varepsilon}(x) \leq \int_{\mathbb{R}^{n}}\left(\int_{0}^{\infty}|F(y, t)|^{2} \frac{d t}{t^{3}}\right)^{1 / 2} \varphi_{\varepsilon}(y) d y=K f * \varphi_{\varepsilon}(x)
$$

Hence

$$
\left\|\nabla f_{\varepsilon}\right\|_{p} \approx\left\|K f_{\varepsilon}\right\|_{p} \leq\|K f\|_{p}\left\|\varphi_{\varepsilon}\right\|_{1}=\|K f\|_{p}<\infty
$$

Since the functions $\nabla f_{\varepsilon}$ are bounded in $L^{p}$, there is a sequence $\varepsilon_{k} \downarrow 0$ such that the sequence $\nabla f_{\varepsilon_{k}}$ converges weakly in $L^{p}$. Since $f_{\varepsilon_{k}} \rightarrow f$ in $L^{p}$ we conclude that $f \in W^{1, p}$. The proof is complete.

\section{Weighted Sobolev SPaces}

In this section we will show that the claims of Theorems 1.4 and 1.6 remain valid in the weighted Sobolev space $W_{w}^{1, p}\left(\mathbb{R}^{n}\right), 1<p<\infty$, where $w \in A_{p}$ as a Muckenhoupt weight; see [9, 11] for the theory of Muckenhoupt weights and [19, Chapter 1] for the theory of weighted Sobolev spaces. We will write $L_{w}^{p}$ or $L^{p}(w)$ to denote the weighted $L^{p}$ space.

Theorem 4.1. Let $w \in A_{p}, 1<p<\infty$, and let $f \in L_{w}^{p}\left(\mathbb{R}^{n}\right)$. Then the following conditions are equivalent

(1) $f \in W_{w}^{1, p}\left(\mathbb{R}^{n}\right)$,

(2) $T f \in L_{w}^{p}\left(\mathbb{R}^{n}\right)$,

(3) $S f \in L_{w}^{p}\left(\mathbb{R}^{n}\right)$.

Moreover for $f \in W_{w}^{1, p}\left(\mathbb{R}^{n}\right)$ we have $\|T f\|_{L_{w}^{p}} \approx\|S f\|_{L_{w}^{p}} \approx\|\nabla f\|_{L_{w}^{p}}$. 
Proof. First of all observe that for $w \in A_{p}, W_{w}^{1, p}\left(\mathbb{R}^{n}\right) \subset W_{\text {loc }}^{1,1}\left(\mathbb{R}^{n}\right)$ (see [19, p.14]) and hence Lemmas 1.5 and 1.7 remain valid in the weighted Sobolev space $W_{w}^{1, p}\left(\mathbb{R}^{n}\right)$.

The proof is based on the following result of Sato [24, Corollary 1] which is a generalization of Theorem 1.2.

Theorem 4.2. For $\varepsilon>0$ let

$$
\zeta(x)=|x|^{-n+\varepsilon} \Omega\left(x^{\prime}\right) \chi_{B(0,1)}(x),
$$

where $x^{\prime}=x /|x|, \Omega \in L^{\infty}\left(S^{n-1}\right)$, and $\int_{S^{n-1}} \Omega(x) d \sigma(x)=0$. Then the square function

$$
\sigma(f)(x)=\left(\int_{0}^{\infty}\left|\zeta_{t} * f(x)\right|^{2} \frac{d t}{t}\right)^{1 / 2}
$$

is bounded in the weighted space $L_{w}^{p}\left(\mathbb{R}^{n}\right), w \in A_{p}, 1<p<\infty$,

$$
\|\sigma(f)\|_{L_{w}^{p}} \leq C_{p, w}\|f\|_{L_{w}^{p}}
$$

As an immediate application we obtain that if $g \in L_{w}^{p}\left(\mathbb{R}^{n}\right)$, then

$$
\|\tilde{T} g\|_{L_{w}^{p}} \leq C\|g\|_{L_{w}^{p}}, \quad\|\tilde{S} g\|_{L_{w}^{p}} \leq C\|g\|_{L_{w}^{p}} .
$$

Indeed, the functions $\phi$ and $\eta$ (defined in (3.1)) satisfy the assumptions of Theorem 4.2. Since $\psi=\phi-\eta$ (see (3.1)) boundedness of the Riesz transform in $L_{w}^{p}$ (see [9, Chapter IV.3],[11]) yields (4.1). It is also easy to see that the duality argument works in the weighted case too. Indeed, let $q$ be the Hölder conjugate exponent to $p$. It directly follows from the definition of the Muckenhoupt weight that $w^{-q / p} \in A_{q}$. Hence the unweighted isometry, Lemma 3.2 yields

$$
\begin{aligned}
C_{1}^{2} \int_{\mathbb{R}^{n}} g h d x & \leq \int_{\mathbb{R}^{n}}\|K g\|_{H}\|K h\|_{H} d x \\
& \leq\left(\int_{\mathbb{R}^{n}}\|K g\|_{H}^{p} w d x\right)^{1 / p}\left(\int_{\mathbb{R}^{n}}\|K h\|_{H}^{q} w^{-q / p} d x\right)^{1 / p} \\
& =\|\tilde{T} g\|_{L_{w}^{p}}\|\tilde{T} h\|_{L^{q}\left(w^{-q / p}\right)} \leq C\|\tilde{T} g\|_{L_{w}^{p}}\|h\|_{L^{q}\left(w^{-q / p}\right)} .
\end{aligned}
$$

Taking the supremum over $\|h\|_{L^{q}\left(w^{-q / p}\right)} \leq 1$ we get

$$
\begin{aligned}
\|g\|_{L_{w}^{p}} & =\left\|g w^{1 / p}\right\|_{p}=\sup _{\left\|h w^{-1 / p}\right\|_{q} \leq 1} \int_{\mathbb{R}^{n}} g w^{1 / p} h w^{-1 / p} d x \\
& =\sup _{\|h\|_{L^{q}\left(w^{-q / p}\right)} \leq 1} \int_{\mathbb{R}^{n}} g h \leq C\|\tilde{T} g\|_{L_{w}^{p}} .
\end{aligned}
$$

A similar argument applies also to $\tilde{S} g$. This combined with (4.1) yields that

$$
\|\tilde{T} g\|_{L_{w}^{p}} \approx\|\tilde{S} g\|_{L_{w}^{p}} \approx\|g\|_{L_{w}^{p}}
$$

Since the Riesz transform is bounded in $L_{w}^{p}$, Lemma 3.6 is also true if we replace $L^{p}$ by $L_{w}^{p}$. Hence as in the unweighted case for $f \in W_{w}^{1, p}$ we have

$$
\|T f\|_{L_{w}^{p}} \approx\|S f\|_{L_{w}^{p}} \approx\|\nabla f\|_{L_{w}^{p}} .
$$

Finally the argument used to show that if $f \in L^{p}$ and $T f \in L^{p}$ or $S f \in L^{p}$, then $f \in W^{1, p}$ also extends to the weighted case, but we need to use boundedness of the Hardy-Littlewood maximal function $\mathcal{M}$ in $L_{w}^{p}$ (see [9],[11, Theorem 9.1.9]). 
Suppose that $f \in L_{w}^{p}$ and $T f \in L_{w}^{p}$ or $S f \in L_{w}^{p}$. We need to show that $f \in W_{w}^{1, p}$. Since $L_{w}^{p} \subset L_{\text {loc }}^{1}, f_{\varepsilon}=f * \varphi_{\varepsilon} \rightarrow f$ a.e. It is easy to see that $\left|f_{\varepsilon}\right| \leq C \mathcal{M} f \in L_{w}^{p}$, and hence the dominated convergence theorem yields that $f_{\varepsilon} \rightarrow f$ in $L_{w}^{p}$. Moreover

$$
\left|\nabla f_{\varepsilon}\right|=\left|f * \nabla \varphi_{\varepsilon}\right| \leq C \varepsilon^{-1} \mathcal{M} f \in L_{w}^{p},
$$

so $f_{\varepsilon} \in W_{w}^{1, p}$. Accordingly $\left\|K f_{\varepsilon}\right\|_{L_{w}^{p}} \approx\|\nabla f\|_{L_{w}^{p}}$. From the proof in the unweighted case we have

$$
\begin{aligned}
& K f_{\varepsilon} \leq K f * \varphi_{\varepsilon} \leq C \mathcal{M}(K f) \in L_{w}^{p}, \\
&\left\|\nabla f_{\varepsilon}\right\|_{L_{w}^{p}} \approx\left\|K f_{\varepsilon}\right\|_{L_{w}^{p}} \leq C\|\mathcal{M}(K f)\|_{L_{w}^{p}}<\infty .
\end{aligned}
$$

Thus $f_{\varepsilon}$ is a bounded family in the reflexive Sobolev space $W_{w}^{1, p}$ (see [19, p.13] for the proof of reflexivity). Since $f_{\varepsilon} \rightarrow f$ in $L_{w}^{p}$, a weak compactness argument implies that $f \in W_{w}^{1, p}\left(\mathbb{R}^{n}\right)$. The proof is complete.

\section{Proof of Proposition 1.9}

Integration by parts gives

$$
\begin{aligned}
\omega_{n}^{2} \int_{\varepsilon}^{T}\left|f(x)-f_{B(x, t)}\right|^{2} \frac{d t}{t^{3}} & =\int_{\varepsilon}^{T}\left(\int_{B(x, t)}(f(x)-f(y)) d y\right)^{2} \frac{d t}{t^{2 n+3}} \\
& =\left.\frac{t^{-2 n-2}}{-2 n-2}\left(\int_{B(x, t)}(f(x)-f(y)) d y\right)^{2}\right|_{\varepsilon} ^{T} \\
& +\frac{1}{2 n+2} \int_{\varepsilon}^{T} t^{-2 n-2} \frac{d}{d t}\left(\int_{B(x, t)}(f(x)-f(y)) d y\right)^{2} d t \\
& =\left.A(t)\right|_{\varepsilon} ^{T}+B_{\varepsilon, T} .
\end{aligned}
$$

We have

$$
\begin{aligned}
B_{\varepsilon, T} & =\frac{1}{2 n+2} \int_{\varepsilon}^{T} t^{-2 n-2} \cdot 2\left(\int_{B(x, t)}(f(x)-f(y)) d y\right)\left(\int_{S(x, t)}(f(x)-f(y)) d \sigma(y)\right) d t \\
& =\frac{2 n \omega_{n}^{2}}{2 n+2} \int_{\varepsilon}^{T}\left(f(x)-f_{B(x, t)}\right)\left(f(x)-f_{S(x, t)}\right) \frac{d t}{t^{3}} \\
& \leq \frac{n \omega_{n}^{2}}{2 n+2}\left[\int_{\varepsilon}^{T}\left|f(x)-f_{B(x, t)}\right|^{2} \frac{d t}{t^{3}}+\int_{\varepsilon}^{T}\left|f(x)-f_{S(x, t)}\right|^{2} \frac{d t}{t^{3}}\right] .
\end{aligned}
$$

In the last step we applied the inequality $a b \leq\left(a^{2}+b^{2}\right) / 2$. Thus

$$
\omega_{n}^{2}\left(1-\frac{n}{2 n+2}\right) \int_{\varepsilon}^{T}\left|f(x)-f_{B(x, t)}\right|^{2} \frac{d t}{t^{3}} \leq\left. A(t)\right|_{\varepsilon} ^{T}+\frac{n \omega_{n}^{2}}{2 n+2} \int_{\varepsilon}^{T}\left|f(x)-f_{S(x, t)}\right|^{2} \frac{d t}{t^{3}}
$$

and it suffices to prove that for almost all $x$,

$$
\left.A(t)\right|_{\varepsilon} ^{T}=A(T)-A(\varepsilon) \rightarrow 0 \quad \text { as } T \rightarrow \infty \text { and } \varepsilon \rightarrow 0
$$

We have

$$
A(T)=C(n)\left(\frac{1}{T}\left(f(x)-f_{B(x, T)} f(y) d y\right)\right)^{2} \rightarrow 0 \quad \text { as } T \rightarrow \infty \text { for almost all } x \in \mathbb{R}^{n} .
$$


Since

we have

$$
\int_{B(x, \varepsilon)} \nabla f(x) \cdot(y-x) d y=0
$$

$$
A(\varepsilon)=C(n)\left(\frac{1}{\varepsilon} f_{B(x, \varepsilon)}(f(x)+\nabla f(x) \cdot(y-x)-f(y)) d y\right)^{2} \rightarrow 0 \quad \text { as } \varepsilon \rightarrow 0
$$

for almost all $x \in \mathbb{R}^{n}$ by [으, Section 6.1.2, Theorem 2].

\section{FinAL REMARKS AND COMMENTS}

6.1. Sobolev spaces on metric spaces. As was pointed out by Alabern, Mateu and Verdera [2], the characterization of $W^{1, p}$ given in Theorem 1.6 can be used to define a Sobolev space on any metric-measure space. It is an interesting question to see how this definition is related to other definitions existing in the literature, [5, 13, 14, 15, 25]. Formally one could try to use the characterization given in Theorem 1.4 to define a Sobolev space on a metric-measure space, but the main difficulty would be that, in general, there is no reasonable way to define measure on the boundary of a ball which would be needed for the spherical averages. It may even happen that the boundary of a ball is empty.

6.2. The spherical maximal function. Kinnunen [20] proved that the HardyLittlewood maximal function is bounded in the Sobolev space, $\mathcal{M}: W^{1, p}\left(\mathbb{R}^{n}\right) \rightarrow W^{1, p}\left(\mathbb{R}^{n}\right)$, $1<p<\infty$. Actually, any sub-linear operator that commutes with translations and is bounded in $L^{p}, 1<p<\infty$ is also bounded in $W^{1, p}$, see e.g. [18, Theorem 1]. From this result it follows that the spherical maximal operator

$$
\mathscr{S} f(x)=\sup _{t>0}\left|f_{S(x, t)} f(y) d \sigma(y)\right|
$$

is bounded in $W^{1, p}$ for $p>n /(n-1)$. Indeed, according to a celebrated result of Stein [29], and Bourgain [4], $\mathscr{S}: L^{p}\left(\mathbb{R}^{n}\right) \rightarrow L^{p}\left(\mathbb{R}^{n}\right)$ is bounded for $p>n /(n-1)$. It was conjectured in [18] that in the range $1<p \leq n /(n-1)$ the spherical maximal operator is a bounded operator from $W^{1, p}$ to the homogeneous Sobolev space $\dot{W}^{1, p}$; see [16, 17] for results supporting this conjecture.

The next result which is a direct consequence of Lemma 2.1 provides another support for this conjecture as it allows to represent $\mathscr{S} f$ as a Hardy-Littlewood type maximal function.

Theorem 6.1. For $f \in W_{\text {loc }}^{1,1}\left(\mathbb{R}^{n}\right)$ we have

$$
\mathscr{S} f(x)=\sup _{t>0}\left|f_{B(x, t)}\left(f(y)-\frac{1}{n} \nabla f(y) \cdot(x-y)\right) d y\right| \text {. }
$$

\section{REFERENCES}

[1] Al-Salman, A., Al-Qhassem, H., Cheng, L. C., Pan, Y.: $L^{p}$ bounds for the function of Marcinkiewicz. Math. Res. Lett. 9 (2002), 697-700.

[2] Alabern, R., Mateu, J., Verdera, J.: A new characterization of Sobolev spaces on $\mathbb{R}^{n}$ Math. Ann. 354 (2012), 589-626. 
[3] Benedek, A., Calderón, A. P., Panzone, R.: Convolution operators on Banach space valued functions. Proc. Nat. Acad. Sci. U.S.A. 48 (1962), 356-365.

[4] Bourgain, J.: Averages in the plane over convex curves and maximal operators. J. Analyse Math. 47 (1986), 69-85.

[5] Chemger, J.: Differentiability of Lipschitz functions on metric measure spaces. Geom. Funct. Anal. 9 (1999), 428-517.

[6] Dorronsoro, J. R.: A characterization of potential spaces. Proc. Amer. Math. Soc. 95 (1985), 21-31.

[7] Duonndikoetxea, J.: Sharp $L^{p}$ boundedness for a class of square functions. Rev. Mat. Complut. 26 (2013), 535-548.

[8] Evans, L. C., Gariepy, R. F.: Measure theory and fine properties of functions. Studies in Advanced Mathematics. CRC Press, Boca Raton, FL, 1992.

[9] García-Cuerva, J., Rubio de Francia, J. L.: Weighted norm inequalities and related topics. North-Holland Mathematics Studies, 116. Notas de Matemática [Mathematical Notes], 104. NorthHolland Publishing Co., Amsterdam, 1985.

[10] Grafakos, L.: Classical Fourier analysis. Second edition. Graduate Texts in Mathematics, 249. Springer, New York, 2008.

[11] Grafakos, L.: Modern Fourier analysis. Second edition. Graduate Texts in Mathematics, 250. Springer, New York, 2009

[12] Grafakos, L.: Classical and modern Fourier analysis. Pearson Education, Inc., Upper Saddle River, NJ, 2004.

[13] HajŁasz, P.: Sobolev spaces on metric-measure spaces. In: Heat kernels and analysis on manifolds, graphs, and metric spaces (Paris, 2002), 173-218, Contemp. Math., 338, Amer. Math. Soc., Providence, RI, 2003.

[14] HajŁasz, P.: Sobolev spaces on an arbitrary metric space. Potential Anal. 5 (1996), 403-415.

[15] Hajeasz, P., Koskela, P.: Sobolev met Poincaré. Mem. Amer. Math. Soc. 145 (2000), no. 688.

[16] HajŁasz, P., Liu, Z.: Sobolev spaces, Lebesgue points and maximal functions. J. Fixed Point Theory Appl. 13 (2013), 259-269.

[17] HajŁasz, P., LiU, Z.: Maximal potentials, maximal singular integrals and the spherical maximal function. Proc. Amer. Math. Soc. 142 (2014), 3965-3974.

[18] HajŁasz, P., Onninen, J.: On boundedness of maximal functions in Sobolev spaces. Ann. Acad. Sci. Fenn. Math. 29 (2004), 167-176.

[19] Heinonen, J., Kilpeläinen, T., Martio, O.: Nonlinear potential theory of degenerate elliptic equations. Oxford Mathematical Monographs. Oxford Science Publications. The Clarendon Press, Oxford University Press, New York, 1993.

[20] Kinnunen, J.: The Hardy-Littlewood maximal function of a Sobolev function. Israel J. Math., 100 (1997), 117-124.

[21] Marcinkiewicz, J.: Sur quelques integrales de type de Dini, Annales de la Société Polonaise, 17 (1938), 42-50.

[22] Nicolau, A.: Divided Differences, Square Functions and a Law of the Iterated Logarithm. arXiv:1404.1746, Preprint (2014).

[23] Sato, S.: Littlewood-Paley operators and Sobolev spaces. Preprint (2014).

[24] Sato, S.: Remarks on square functions in the Littlewood-Paley theory. Bull. Austral. Math. Soc. 58 (1998), 199-211.

[25] Shanmugalingam, N.: Newtonian spaces: an extension of Sobolev spaces to metric measure spaces. Rev. Mat. Iberoamericana 16 (2000), 243-279.

[26] Stein, E. M.: On the functions of Littlewood-Paley, Lusin, and Marcinkiewicz. Trans. Amer. Math. Soc. 88 (1958), 430-466.

[27] Stein, E. M.: The characterization of functions arising as potentials. Bull. Amer. Math. Soc. 67 (1961), 102-104.

[28] Stein, E. M.: Singular integrals and differentiability properties of functions. Princeton Mathematical Series, No. 30 Princeton University Press, Princeton, N.J. 1970

[29] Stein, E. M.: Maximal functions. I. Spherical means. Proc. Nat. Acad. Sci. U.S.A. 73 (1976), 2174-2175. 
[30] Strichartz, R. S.: Multipliers on fractional Sobolev spaces. J. Math. Mech. 16 (1967), 1031-1060.

[31] Torchinsky, A.: Real-variable methods in harmonic analysis. Pure and Applied Mathematics, 123. Academic Press, Inc., Orlando, FL, 1986.

[32] Waterman, D.: On an integral of Marcinkiewicz. Trans. Amer. Math. Soc. 91 (1959), 129-138.

[33] Wheeden, R. L.: Lebesgue and Lipschitz spaces and integrals of the Marcinkiewicz type. Studia Math. 32 (1969), 73-93.

[34] Wheeden, R. L.: A note on a generalized hypersingular integral. Studia Math. 44 (1972), 17-26.

[35] Zygmund, A.: On certain integrals, Trans. Amer. Math. Soc. 55 (1944), 170-204.

[36] Zygmund, A.: Trigonometric series. Vol. I, II. Third edition. With a foreword by Robert A. Fefferman. Cambridge Mathematical Library. Cambridge University Press, Cambridge, 2002.

P. Hajeasz: Department of Mathematics, University of Pittsburgh, 301 Thackeray Hall, Pittsburgh, PA 15260, USA, hajlasz@pitt.edu

Z. Liu: Department of Mathematics and Statistics, University of Jyväskylä, P.O. Box 35 (MaD), FI-40014 JyväskYlä, Finland, liuzhuomin@hotmail.com 